\title{
Effects of Different Whitening agents on the Mechanical Properties of Restorative Materials
}

\author{
Heba Abdullah Mashat ${ }^{1 *}$, Waad Essa Alsulami ${ }^{2}$ \\ ${ }^{1}$ Independent Researcher, Jeddah KSA \\ ${ }^{2}$ Dental Resident, Ministry of Health, Jeddah KSA
}

DOI: 1 10.36348/SJODR.2019.v04i10.003

| Received: 03.10.2019 | Accepted: 11.10.2019| Published: 17.10.2019

*Corresponding author: Heba Abdullah Mashat

\section{Abstract}

Dental bleaching procedures are commonly performed to improve esthetics. It can be either in-office professional bleaching techniques or self-administered home-based bleaching agents. These bleaching agents contain variable concentrations of hydrogen or carbamide peroxide that initiates oxidation process mechanism which reduces the extrinsic tooth discoloration. The objective of this review was to discuss the influence of different whitening agents on the mechanical properties of restorative materials. Dentists should be aware of the mechanical properties of restorative materials and ideally prepare the patients free of carious lesions before performing the bleaching procedures. However, bleaching agents with new formulations are introduced that doesn't impact the surface integrity of existing restorations. Moreover, composite restorations on bleached enamel surfaces should be placed after at least 2 weeks. For those patients that require restorative treatment immediately, use of antioxidant agents before bonding procedures on bleached enamel neutralizes completely the deleterious effects of bleaching and increases the bond strength.

Key words: bleaching; composite restorations; whitening agents; whitening treatments, tooth bleaching.

Copyright @ 2019: This is an open-access article distributed under the terms of the Creative Commons Attribution license which permits unrestricted use, distribution, and reproduction in any medium for non-commercial use (NonCommercial, or CC-BY-NC) provided the original author and source are credited.

\section{INTRODUCTION}

Dental bleaching procedures are commonly performed to improve esthetics. It can be either inoffice professional bleaching techniques or selfadministered home-based bleaching agents. These bleaching agents contain variable concentrations of hydrogen or carbamide peroxide that initiates oxidation process mechanism which reduces the extrinsic tooth discoloration. Some studies have reported the repeatedly use of these whitening agents or if they are applied for longer periods might cause some adverse effects on the tooth structure and extra coronal restorations. These adverse effects include softening, roughness, cracks and invisible color changes. Some investigators have discussed that bleaching agents might induce increase surface roughness of composite and porcelain materials while others reported that low concentrations of home-based whitening gels don't cause any significant changes in the surface integrity of extra coronal restorations. Nevertheless, novel whitening formulations derived from the natural materials are introduced that might help in reducing the drawbacks associated with the existing formulas and can produce effective whitening without altering the tooth structures and restorative materials [1].
With the advancement in technology, new methods are invented to measure the mechanical properties of both dental tissues and restorative materials. Nanoindentation method is used to calculate the mechanical properties in extremely thin regions such as the tooth-restoration interface unlike conventional microhardness testing procedures such as scanning electron microscopy (SEM) analysis. Therefore, this method can be implemented to evaluate the effects of bleaching agents on the existing restorations [2].

The objective of this review was to discuss the influence of different whitening agents on the mechanical properties of restorative materials.

\section{Overview}

Numerous studies have discussed the effects of bleaching agents regardless of the techniques on the surface integrity and physical properties of dental restorative materials.

\section{Novel Formulations in Bleaching Agents}

A study by Firoozmand LM et al. investigated the in vitro bond strength of composite resins on enamel previously treated with the whitening strips. A total of 
48 bovine incisors were divided into four experimental groups and treated with different whitening strips containing variable concentrations of hydrogen peroxides $(9.5 \%, 10 \%$ and $7.5 \%$ with Fluorine, calcium, and potassium nitrate content) respectively. The specimens were subjected to a whitening procedure for over 2 weeks. Moreover, significant differences were evident among the groups $(\mathrm{p}<0.05)$. Authors concluded that new whitening formulation containing a gel with $7.5 \%$ hydrogen peroxide with added calcium and fluorine increases the bond strength of composite restorations as compared the other bleaching strips that decreased the bond strength following 14 days of treatment [3].

On the other hand, a study evaluated the effects of two different whitening strips on color, microstructure, and roughness of tea-stained porcelain and composite surfaces. 54 porcelain and 72 composite chins served as the specimen and treated with a novel (Crest ${ }^{\circledR}$ 3D White Whitestrips ${ }^{\mathrm{TM}}$ and Oral Essentials ${ }^{\circledR}$ Whitening Strips) or control dental whitening strips. The sample surfaces were examined by light microscopy, profilometry, and SEM. No discrete physical defects were seen in the light microscopy and SEM images whereas high-resolution SEM and profilometry showed an appearance of increased surface roughness in all composite samples. Hence, a novel whitening strip demonstrated a comparable whitening effect and didn't induce physical defects or roughening of the composite sample surfaces [1].

\section{Influence of Bleaching Agents on the Mechanical Properties}

In-office bleaching procedure has some benefits over the other home-based bleaching techniques. However, some studies have reported that laser-assisted and conventional in-office bleaching might interact with the single or double c-c bonds and can degrade the three-dimensional polymer network of dental composites. This would cause dimensional changes, influences the mechanical properties and affects the clinical durability of restorations. A study by Omarani et al. demonstrated the effects of In-office bleaching techniques on monomer release from microhybrid and nanohybrid composite. 2 samples of each composite type was subdivided into 4 groups and treated with 1: Conventional in-office bleaching (CIB) using Opalescence Boost PF 38\% gel, 2: Laser-assisted bleaching (LBO) using Opalescence Boost PF 38\% gel, 3: Laser-assisted bleaching ( $\mathrm{LBH}$ ) with the JW Power bleaching gel, 4: (CO) control without bleaching respectively. Hence, nanohybrid composites showed significantly more monomer release than microhybrid composites $(\mathrm{P}<0.05)$. Bleaching by laser with JW Power Bleaching gel can significantly lead to more monomer release in nanohybrid composite [4]. While an in vitro study revealed bleaching with $35 \%$ hydrogen peroxide gel did not enhance the surface roughness of nanohybrid composite [5].
A study was conducted to evaluate the influence of three different whitening systems (opalescence 10\%, whiteness perfect $16 \%$, and whiteness HP 35\%) on the microtensile bond strength $(\mu \mathrm{TBS})$ of resin composite to bleached enamel at different time intervals after the bleaching procedure was performed. Authors suggested that adhesive restorations should not be placed immediately after 1 week irrespective of the type or concentration of the whitening system used. Composite restorations on bleached enamel surfaces should be placed after at least 2 weeks [6]. On the other hand; bleaching agents (15\% carbamide peroxide, $35 \%$ hydrogen peroxide and lightactivated $35 \%$ hydrogen peroxide) significantly decreased the microhardness of a silorane-based composite resin [7].

Another study supported that all carbamide peroxide bleaching agent with variable concentrations $(10 \%, 16 \%$, or $22 \%)$ significantly decreased the microhardness of the methacrylate-based composite resin groups, while microhardness was significantly increased in the silorane-based composite resin groups [8]. The surface microhardness of the composite restorative materials mainly depends on the composition. The use of Er, Cr: YSGG laser during inoffice tooth whitening procedure did not affect the surface microhardness and roughness of the composite restorative materials. Using a high concentrated inoffice bleaching agent with $40 \%$ hydrogen peroxide decreased the surface microhardness of the viscous or flowable composite restorative materials [9].

Moreover, office-based bleaching agent containing $45 \%$ carbamide peroxide was observed to be more secure for composite resin than $38 \%$ hydrogen peroxide. Home bleaching agents despite the low concentrations can cause a significant decrease in the surface hardness of composite resins as their application time is longer [10]. A study by Esra et al. also suggested that home bleaching agents increased the surface roughness of all composite resins [11].

On the other hand, a study reported that application of different whitening systems does not have any adverse effect on the Flexure strength (FS) of hybrid composite resins. Moreover, in-office bleaching agents with hydrogen peroxide might have a lesser impact on the FS. 12

A study supported that no remarkable difference was observed between the bleaching agents either Opalescence Boost or Whiteness Hydrogen peroxide Blue for the surface roughness of composite and porcelain crowns. The whitening agents can be applied without affecting the ceramic, nano filled or nanohybrid resin restorations. Moreover, bleaching might damage the surface finish of the microhybrid or microfilled composite restorations [13] Nevertheless, 
tooth bleaching does not damage the tooth-restoration interface of composite restorations [14].

Moreover, the stability of bonded resin composite restorations to enamel with the use of different dental adhesives could be compromised after the bleaching procedure. Therefore, replacement of bonded resin composite restorations might be considered especially if these restorations were bonded previously with self-etch adhesives [15].

\section{Additional Clinical Interventions}

Some studies have suggested that the negative effects of whitening agents on composite bonding can be neutralized by applying $35 \%$ sodium ascorbate for two minutes before placing composite restorations. This technique is clinically relevant and applicable for those patients that require restorative treatment immediately after in-office bleaching techniques [16]. Moreover, use of antioxidant (10\% sodium ascorbate gel or $5 \%$ proanthocyanidin agent) before bonding procedures on bleached enamel neutralizes completely the deleterious effects of bleaching and significantly increases the bond strength [17].

Another study suggested that the use of antioxidant agents irrespective of their type, form, concentration and the duration of application use can improve the surface bond strength (SBS) of bleached enamel. Therefore, antioxidant treatment can be considered an appropriate technique for enhancing the SBS of bleached teeth [18].

Whitening procedure is known to decrease biofilm formation. A study by Mahmoud Bahari et al. discussed that lowest biofilm formation was reported in the group subjected to light-activated group with $35 \%$ hydrogen peroxide relative to other bleaching agents [19].

\section{CONCLUSION}

Dentists should be aware of the mechanical properties of restorative materials and ideally prepare the patients free of carious lesions before performing the bleaching procedures. However, bleaching agents with new formulations are introduced that doesn't impact the surface integrity of existing restorations. Moreover, composite restorations on bleached enamel surfaces should be placed after at least 2 weeks. For those patients that require restorative treatment immediately, use of antioxidant agents before bonding procedures on bleached enamel neutralizes completely the deleterious effects of bleaching and increases the bond strength.

\section{REFERENCES}

1. Takesh, T., Sargsyan, A., Lee, M., Anbarani, A., Ho, J., \& Wilder-Smith, P. (2017). Evaluating the Whitening and Microstructural Effects of a Novel
Whitening Strip on Porcelain and Composite Dental Materials. Dentistry (Sunnyvale, Calif.), 7(8).

2. Abe, A. T., Youssef, M. N., \& Turbino, M. L. (2016). Effect of bleaching agents on the nanohardness of tooth enamel, composite resin, and the tooth-restoration interface. Operative dentistry, 41(1), 44-52.

3. Firoozmand, L. M., Reis, W. L., Vieira, M. A., Nunes, A. G., Tavarez, R. R., Tonetto, M. R., ... \& Bandeca, M. C. (2015). Can Whitening Strips interfere with the Bond Strength of Composite Resins?. The journal of contemporary dental practice, 16(4), 259-263.

4. Omrani, L. R., Farjadfar, S., Pedram, P., Sadray, S., Kamangar, S. S. H., \& Chiniforoush, N. (2017). Effect of laser-assisted and conventional in-office bleaching on monomer release from microhybrid and nanohybrid composite. Laser therapy, 26(2), 89-96.

5. Halacoglu, D. M., Yamanel, K., Basaran, S., Tuncer, D., \& Celik, C. (2016). Effects of staining and bleaching on a nanohybrid composite with or without surface sealant. European journal of dentistry, 10(3), 361.

6. Topcu, F. T., Erdemir, U., Ozel, E., Tiryaki, M., Oktay, E. A., \& Yildiz, E. (2017). Influence of bleaching regimen and time elapsed on microtensile bond strength of resin composite to enamel. Contemporary clinical dentistry, 8(3), 451.

7. Bahari, M., Oskoee, S. S., Mohammadi, N., Chaharom, M. E. E., Godrati, M., \& Oskoee, A. S. (2016). Effect of different bleaching strategies on microhardness of a silorane-based composite resin. Journal of dental research, dental clinics, dental prospects, 10(4), 213.

8. Esmaeili, B., Abolghasemzadeh, F., Gholampor, A., \& Daryakenari, G. (2018). The effect of home bleaching carbamide peroxide concentration on the microhardness of dental composite resins. General dentistry, 66(1), 40-44.

9. Dionysopoulos, D., Strakas, D., Tsitrou, E., Tolidis, K., \& Koumpia, E. (2016). Effect of Er, Cr: YSGG laser on the surface of composite restoratives during in-office tooth bleaching. Lasers in medical science, 31(5), 875882.

10. Yikilgan, İ., Kamak, H., Akgul, S., Ozcan, S., \& Bala, O. (2017). Effects of three different bleaching agents on microhardness and roughness of composite sample surfaces finished with different polishing techniques. Journal of clinical and experimental dentistry, 9(3), e460.

11. Cengiz, E., Kurtulmus-Yilmaz, S., Ulusoy, N., Deniz, S. T., \& Yuksel-Devrim, E. (2016). The effect of home bleaching agents on the surface roughness of five different composite resins: A SEM evaluation. Scanning, 38(3), 277-283.

12. Feiz, A., Samanian, N., Davoudi, A., \& Badrian, H. (2016). Effect of different bleaching regimens on 
Heba Abdullah Mashat \& Waad Essa Alsulami; Saudi J Oral Dent Res, Oct 2019; 4(10): 700-703

the flexural strength of hybrid composite resin. Journal of conservative dentistry: JCD, 19(2), 157.

13. Qasim, S., Ramakrishnaiah, R., Alkheriaf, A. A., \& Zafar, M. S. (2016). Influence of various bleaching regimes on surface roughness of resin composite and ceramic dental biomaterials. Technology and Health Care, 24(2), 153-161.

14. Silva, L., Thedei Jr, G., Menezes-Oliveira, M. A., Nogueira, R. D., \& Geraldo-Martins, V. (2017). Tooth bleaching effects on the adhesive interface of composite restorations. The international journal of esthetic dentistry, 12(1), 96-106.

15. El Mourad, A. M. (2019). Stability of Bonded Resin Composite Restorations to Enamel after Bleaching with 20\% Carbamide Peroxide. The journal of contemporary dental practice,20(2), 247.

16. Ismail, E. H., Kilinc, E., Hardigan, P. C., Rothrock, J. K., Thompson, J. Y., \& Garcia-Godoy, C. (2017). Effect of two-minute application of $35 \%$ sodium ascorbate on composite bond strength following bleaching. The Journal of Contemporary Dental Practice electronic resource, 18(10), 874.

17. Manoharan, M., Shashibhushan, K. K., Poornima, P., Naik, S. N., Patil, D., \& Shruthi, A. S. (2016). Effect of newer antioxidants on the bond strength of composite on bleached enamel. Journal of Indian Society of Pedodontics and Preventive Dentistry, 34(4), 391.

18. Feiz, A., Mosleh, H., \& Nazeri, R. (2017). Evaluating the effect of antioxidant agents on shear bond strength of tooth-colored restorative materials after bleaching: A systematic review. Journal of the mechanical behavior of biomedical materials, 71, 156-164.

19. Bahari, M., Chaharom, M. E. E., Daneshpooy, M., Gholizadeh, S., \& Pashayi, H. (2019). Effect of bleaching protocols on surface roughness and biofilm formation on silorane-based composite resin. Dental Research Journal, 16(4), 264. 